\title{
A Low-Overhead Hybrid Routing Algorithm for ZigBee Networks
}

\author{
Zhi Ren, Lihua Tian, Jianling Cao, Jibi Li, Zilong Zhang \\ Chongqing Key Lab of Mobile Communications Technology, Chongqing University of Posts and \\ Telecommunications, Chongqing, China, 400065
}

Email: tianlihua7987@163.com

Keywords: ZigBee networks; optimal paths; overhead; residual energy

\begin{abstract}
In order to improve the non-optimal paths worked out by the ZigBee cluster-tree routing (ZTR) algorithm and to reduce the overhead of the AODVjr algorithm, we propose in this paper a low-overhead hybrid routing algorithm (LOHRA) for ZigBee networks. LOHRA effectively limits the flooding range of RREQ through utilizing the descendents of nodes and considers the length of paths and the minimum residual energy in path as routing metrics. Therefore, it not only ensures that the newly built paths are better than those of ZTR, but also reduces the redundant overhead of AODVjr and postpones the death of the nodes with low energy. Theoretical analysis and simulation results show that LOHRA outperforms the classical ZTR algorithm and AODVjr algorithm in terms of network overhead, network lifetime, and the end-to-end delay of data packets.
\end{abstract}

\section{Introduction}

ZigBee is a wireless networking technology which has a low data baud rate within short distances at low cost and power consumption based on the ZigBee standard[1], which is mainly used for short distance and low rate data transmission between various kinds of electronic equipment, and low reaction time, periodic/ intermittent data transmission occasions.

ZigBee specification defines two routing protocols: ZigBee tree routing (ZTR) and AODVjr routing protocol[1]. ZTR is especially suitable for cluster tree network topology and applied in conjunction with Distributed Address Assignment Mechanism (DAAM)[1].he node determines to forward the data to the parent or child node along the tree structure through calculating without routing table. However, ZTR often provides inefficient paths with more transmission delays [2]. AODVjr [3] can find the optimal path through flooding RREQ. However the flooding mechanism has high control overhead and significantly wastes amount of bandwidth, memory and device energy. To address these issues, we propose a lower overhead hybrid routing algorithm which reduces the RREQ forwarding while keeping path discovery results. Meanwhile, consider the length of path and residual energy of nodes, LOHRA can save energy consumption of nodes and prolong network lifetime.

The rest of the paper is organized as follows: Technical background and related work are introduced in section2. Section 3 details LOHRA. Simulation results are shown and analyzed in section4. Finally, section 5 gives conclusions.

\section{Related Works}

In ZTR algorithm, the addresses are based on DAAM and the nodes will send data packets to the parent or child node. ZTR does not need discover route and store any routing information, so it is easily complement. However, ZTR cannot ensure the optimal path and the transmission delay may be high.

To overcome the disadvantages of ZTR, researchers have put forward some improvements [4-6] in recent years. The neighbor nodes are used to reduce the path length, communication overhead and transmit delay in [4][5]. Yang et al.[6] propose Improved Enhanced Tree Routing algorithm, only the neighbors whose residual energy are above the energy threshold can undertake the packet forwarding tasks, so decreases the death number of nodes and prolongs the network lifetime.

In the field of AODVjr algorithm, Lin et al. [7]propose LF-ZAODV algorithm, it uses the hops of ZTR path-1 limiting RREQ forward range and reduces the flooding overhead, but ignores the energy 
consumption. Wang Fang [8] conduct an improved algorithm which was based on the role differences and current energy state of the node to overcome the high cost of the traditional AODVjr. E-MAODVjr algorithm is presented in [9], the destinations choose the least link loss path reply RREP, and establish the route, thus balance node energy consumption.

In conclusion, the existing routing algorithms for ZigBee networks still exist high overhead as redundancy RREQ, and the routing metric is not optimal, so a further study is required.

\section{LOHRA Algorithm}

To overcome the flaws of non-optimal path of ZTR and the high overhead of AODVjr, we propose LOHRA for ZigBee Networks in this paper. LOHRA reduces the flooding of RREQ through limiting the broadcast range, and sets the new routing metric to selects the shortest path with more residual energy, thus it not only ensures the new built path is better than ZTR, but also reduces the redundant overhead of AODVjr and avoids the failure of the node with low energy, thus LOHRA can efficiently save energy of nodes and prolong the network lifetime.

Steps of LOHRA. LOHRA implements after the network has used DAAM design addresses. The destinations are randomly set in the network. The processes of LOHRA are as follows:

Step1. The source first determines if the destination is in its routing table, and if so, sends the data to next hop according to routing table. Otherwise, executes step2.

Step2. The source initiates and sends a RREQ, the set of hop threshold is $H_{T R^{-}} 1$.

$H_{T R}=D s+D d-2 D p$.

where $D s$ and $D d$ represent the depth of the source and destination, $D p$ is the depth of large depth common ancestor of the source and destination. If the source doesn't receive RREP in a schedule time, it will forward data by ZTR. If received, forward data according the new built path.

Step3. The intermediate router processes RREQ:

i. If the RREQ has been received or past hops is over threshold, discards it. Otherwise, performs ii.

ii. The node calculates whether the current node is one of the descendents of the large depth common ancestor of the source and destination. The calculated formula as follows:

$A p<A<A p+C \operatorname{sip}(D p-1)$.

where $A$ is the network address of the current node, $A p$ and $D p$ are the network address and depth of the large depth common ancestor of the source and destination.

If so, updates the TTL of RREQ, and broadcasts RREQ. Otherwise, discards RREQ.

Step4. The destination receives RREQ. If the number of experienced hops is less than the threshold, it responds RREP to the source along the reverse direction. The RREP contains the minimum residual energy of nodes in path (initial value is residual energy of the destination).

Step5. The intermediate node receives RREP, establishes the route to the destination, and updates the value of relevant field in RREP, then unicasts RREP to the source.

Step6. The source receives RREP and establishes the route table, and storages the integrated hops.

Limiting the range of flooding RREQ. LOHRA limits the flooding range of RREQ through utilizing the descendents of nodes.

As DAAM is based on the tree structure and it correlates the network address with the position information through the parent-child relationship. Therefore, the nodes which have the same ancestor will form a cluster. If the source and destination are in the same cluster, the nodes in the same cluster will mostly provide the optimal path. Making use of these, only the descendents of the large depth common ancestor of the source and destination forward RREQ, thus LOHRA limits the flooding range and reduces overhead while keeping path discovery results.

A New Routing Metric-Integrated Hops. In LOHRA, we propose a new routing metricIntegrated Hops. When the source receives RREPs from multiple paths, the node will choose the path which is shortest and has more residual energy. Integrated Hops is defined as follows:

$$
h_{i}=h+\frac{E_{i}-E_{r}}{E_{i}} .
$$


where $h$ is the hops to destination of the path, Ei and $E r$ are the initial energy and the minimum residual energy of the nodes in the path respectively.

Lemma1. Integrated Hops in turn considers the length of path, the minimum residual energy of path.

Proof: According to the formula (3), we obtain, $h \geq 1$,

$\because 0<E_{r} \leq E_{i} \Rightarrow E_{i}-E_{r}<E_{i} \quad \therefore \frac{E_{i}-E_{r}}{E_{i}}<1 . \quad$ The proof is completed.

\section{Performance Evaluation}

In this section we conduct simulations to evaluate the performance of LOHRA against that of ZTR and AODVjr. Simulations are realized using OPNET14.5.

In our performance evaluation, we considered the following metrics:

(1) The normalized network overhead, the ratio of the bits of all nodes transmit packets (include control and data packets) to the bits of all successfully received data packets.

(2) Network lifetime, the total time during which the network is alive.

(3) Average end-to-end delay, the average time for all surviving data packets transmission.

Normalized network overhead. Before nodes die, we test the normalized network overhead. The results are shown in Fig. 1.

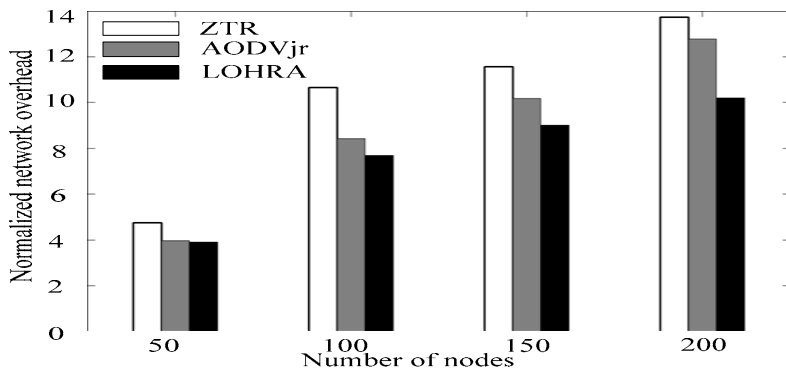

Fig.1 Comparison of normalized network overhead.

Fig. 1 records that LOHRA effectively decreases the network overhead. Due to ZTR can't provide the optimal path, AODVjr broadcasts RREQ in the network, these introduce high overhead. LOHRA shortens the length of path than ZTR and reduces the redundant RREQ forwarding through setting the hop threshold and limiting the broadcast range. Thus LOHRA has the lowest network overhead.

Network lifetime. The energy model refers to [10]. When the residual energy is less than 5\% of the initial energy, the node is failed. And when the proportion of failed nodes is more than $20 \%$, the network becomes not operational. Statistical results are shown in Fig. 2.

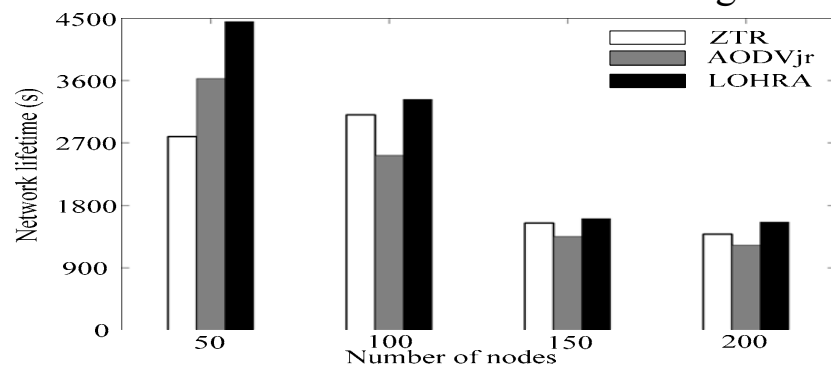

Fig.2 Comparison of network lifetime.

Fig.2 shows that LOHRA extends network lifetime compared with ZTR and AODVjr. In ZTR, the nodes at lower depth consume more energy, and die faster. LOHRA chooses the shortest path to forward date to decrease the task of nodes which adjoin sink. Meanwhile, LOHRA reduces the network overhead and saves the energy consumption of nodes by limiting the hops and RREQ broadcast range. Thus LOHRA prolongs the network lifetime.

Average end-to- end delay. Before nodes die, we measure the average end-to-end delay of the packet delivery. The results are shown in Fig. 3. 
From Fig.3, it could be found that the average end-to-end delay of data packet delivery of LOHRA is lower than ZTR and AODVjr. In ZTR, the nodes are more close to the sink, the higher traffic they have, thus the delay is mainly from packet forward delay and queue delay at the smaller depth nodes. AODVjr and LOHRA can find the shortest path and decrease the packet forward times, so effectively reduce delay. In addition, LOHRA reduces the broadcast of RREQ, the network traffic is lower than AODVjr, so the data can faster arrive at the optimal path.

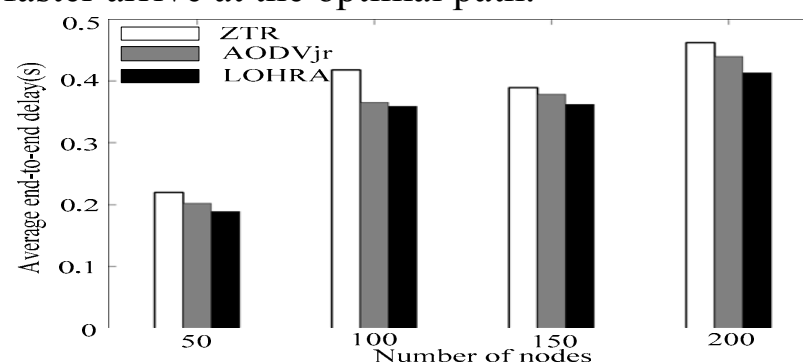

Fig.3 Comparison of average end-to end delay.

\section{Conclusions}

In this paper, we propose the LOHRA algorithm, which reduces the redundant RREQ forwarding by setting the hops threshold and limiting the broadcast range when creates route. Meanwhile, sets the path length and residual energy of nodes as the routing metrics, achieves reducing the network overhead. Theoretical analysis and simulation results show that our proposed algorithm outperforms ZTR and AODVjr. Our future work includes continuing to reduce network overhead and nodes' energy consumption based on LOHRA, and improving the network performance on the whole.

\section{Acknowledgments}

This work was supported in part by National Natural Science Foundation of China (60972068), the Scientific Research Starting Foundation for Returned Overseas Chinese Scholars, Ministry of Education of China (2010-1561), the Science Research Project of Education Committee of Chongqing (KJ120510), the open project of Emergency Communication Laboratory of Chongqing (201201), and the transformation project of excellent achievement of Chongqing Municipal Education Commission (Kjzh11206).

\section{References}

[1] ZigBee Alliance. Document 053474r17, ZigBee Specification, 2007: 370-372.

[2] X.-Q. Zhu, Z.-X. Chen. Design of Design of Home Wireless Network Using Routing Algorithm of Tree and AODVjr-PB [J]. Journal of Chongqing University of Posts and Telecommunications, 2011, 21(3): 343-348.

[3] I. D. Chakeres, L. Klein-verndt. AODVjr, AODV Simplified [J]. Mobile Computing and Communication Review, 2002, 6(3): 100-101.

[4] T. Kim, D. Kim, N. Park. Shortcut Tree Routing in ZigBee Networks[C]. IEEE Second Wireless Pervasive Computing (ISWPC2007), 2007: 42-47.

[5] J. Y. Ha, H. S. Park, S. Choi, W. H. Kwon. EHRP: Enhanced Hierarchical Routing Protocol for ZigBee Mesh Networks [J]. IEEE Communication Letters, 2007, 11(2): 1028-1030.

[6] G.-S. Yang, Z.-J. Wang, W. Wang. An Improved ETR Protocol with Energy Awareness for Sensor Networks[C]. 2009 Fifth International Conference on Mobile Ad-hoc and Sensor Networks, 2009: 117-123.

[7] Z.-J. Lin, Q.-H. Meng, H.-W. Liang. A Route Discovery Method based on Limited Flooding in ZigBee Networks[C]. IEEE International Conference on Automation and Logistics, Qingdao, 2008: 3039-3044. 
[8] F. Wang, Q.-L. Qiao, Y.-L. Ban. Improved Routing Algorithm for ZigBee Mesh Networks [J]. Computer Applications, 2008, 28(11): 2788-2791.

[9] J. Xiao, X.-J. Liu. The Research of E-AOMDVjr Routing Algorithm in ZigBee Network[C]. 2011 Chinese Control and Decision Conference (CCDC), 2011: 2360-2365.

[10] Y.-Zhang, Y.-Li, Z.-J. Liu. Cluster-head Slection Enhanced Algorithm Based on Energy for Wireless Sensor Networks [J]. Journal of Chongqing University of Posts and Telecommunications, 2007, 19(5): 1-4. 